\title{
Diabetes MODY tipo 5: a propósito de un caso
}

\section{Maturity-onset diabetes of the young type 5: a case report}

\author{
S. Junquera, M. J. Goñi, J. Lafita
}

\section{RESUMEN}

La diabetes MODY-5 es un tipo de diabetes monogénica infrecuente, causada por mutación en el gen del factor de transcripción nuclear hepático 1-beta (HNF$1 \beta)$. Esta mutación puede ser de tipo puntual o bien corresponder a delecciones grandes, y a su vez, puede aparecer de novo por mutación espontánea o bien ser transmitida de forma hereditaria con carácter autosómico dominante. Está asociada con un alto riesgo de complicaciones microvasculares de aparición temprana en las personas afectas, así como con alteraciones renales características del tipo quistes y anomalías del tracto genital, que están presentes incluso antes del nacimiento. Por ello, está justificado hacer pruebas de detección para las mutaciones de HNF-1 $\beta$ en diabéticos no obesos, sobre todo, cuando existen alteraciones renales o genitales asociadas, sin tener en cuenta los antecedentes familiares.

Palabras clave. Diabetes MODY-5.

\begin{abstract}
MODY-5 diabetes is an infrequent type of monogenic diabetes, caused by mutation in the gene of the nuclear hepatic transcription factor 1-beta (HNF-1 $\beta$ ). This mutation can be of a momentary type or it might correspond to big deletions, and, in its turn, it can appear due to spontaneous de novo mutation or it can be transmitted by hereditary with an autosomal dominant character. It is associated with a high risk of microvascular complications that appear early in affected people, as well as with characteristic renal alterations of the cyst type, and anomalies of the genital tract, which are present even before birth. That is why it is justified to carry out detection tests for HNF-1 $\beta$ mutations in non-obese diabetics, above all when there are associated renal or genital alterations, without consideration of family antecedents.
\end{abstract}

Key words. MODY-5 diabetes.
Complejo Hospitalario de Navarra. Servicio de Endocrinología.

Recepción: 9 de agosto de 2011

Aceptación provisional: 5 de septiembre de 2011

Aceptación definitiva: 9 de septiembre de 2011

\section{Correspondencia:}

Sonia Junquera Bañares

Complejo Hospitalario de Navarra

Servicio de Endocrinología

C/ Irunlarrea, 3

31008 Pamplona

E-mail: sojuba@hotmail.com 


\section{INTRODUCCIÓN}

La diabetes MODY tipo 5 constituye una causa infrecuente de diabetes monogénica (1-2\%) y fue descrita en 1997 por primera vez en un sujeto japonés. Este tipo de MODY es de comienzo temprano, antes de 25 años; se asocia a nefropatía no diabética y conlleva un alto riesgo de complicaciones microvasculares que suelen aparecer de forma prematura ${ }^{1-3}$. Está causada por una mutación en el gen que codifica el factor de transcripción HNF-1 $\beta$, situado en el locus del gen 17cen-q21.3. Es de herencia autosómica dominante y tiene una penetrancia del $90-95 \%$. Se expresa en células ductales embrionarias y adultas del páncreas, epitelio genitourinario, vía biliar y pulmón. La función de este factor en las células $\beta$ del páncreas es la de regular la expresión de los genes de la insulina, así como de las proteínas implicadas en el transporte y en el metabolismo mitocondrial (todos ligadas a la secreción de insulina) y al metabolismo de las lipoproteínas. Las personas portadoras de esta mutación tienen defectos en la respuesta secretora de la insulina a una variedad de secretagogos, especialmente la glucosa, que ya aparecen antes del inicio de la hiperglucemia y que denota la existencia de un defecto funcional primario en este síndrome. También se han observado respuestas reducidas al glucagón frente a la arginina, lo que sugiere que esta mutación implica una alteración más extensa del desarrollo pancreático que la célula $\beta$ exclusivamente ${ }^{4}$.

\section{CASO CLÍNICO}

Se trata de una paciente de 25 años, natural de Ucrania, diagnosticada de diabetes secundaria a agenesia pancreática desde hace 5 años, ingresada en nuestro servicio procedente del servicio de Urgencias por una descompensación hiperglucémica cetósica no acidótica. La paciente desde el diagnóstico ha tenido un control metabólico aceptable con HbA1c menores de 7\% con tratamiento dietético y ejercicio.

Entre los antecedentes familiares tiene un bisabuelo diabético y padres y hermanos sanos.

En la anamnesis, al ingreso la paciente refiere mal control metabólico desde hace dos meses, coincidiendo con el inicio de episodios frecuentes de infecciones urinarias, y últimamente, agravado por candidiasis vulvovaginal y paroniquia en dedos de las manos, con clínica de polidipsia, poliuria intensa y pérdida de $6,4 \mathrm{~kg}$ de peso durante este periodo.

En la exploración física destaca: talla $167 \mathrm{~cm}$; peso $38,6 \mathrm{~kg}$; IMC $13,4 \mathrm{~kg} / \mathrm{m} 2$; TA $120 / 80 \mathrm{mmHg}$; FC 116 lpm; saturación 97\%. Mandíbula en farol. Tiroides grado 0. AC y AP normal. Abdomen blando y depresible, no doloroso, sin masas ni megalias. MMII sin edemas y pulsos periféricos conservados. Sensibilidad vibratoria, con monofilamento y reflejos osteotendinosos conservados. Lesiones eritematosas y pruriginosas en región vulvar y perianal sugestivas de candidiasis. Paroniquia en cuarto dedo mano derecha. Dermopatía diabética extensa en piernas y brazos.

Pruebas complementarias: Hemograma normal, VSG $35 \mathrm{~mm} / \mathrm{h}$, glucosa $552 \mathrm{mg} / \mathrm{dL}, \mathrm{Cr} 0,8$ $\mathrm{mg} / \mathrm{mL}$, colesterol total $205 \mathrm{mg} / \mathrm{dL}$, c-LDL 125 $\mathrm{mg} / \mathrm{dL}$, cHDL $58 \mathrm{mg} / \mathrm{dL}$. Perfil hepático normal salvo GOT 54 UI (7-32), GPT 63 UI (5-31) y GGT 59 (7-32). Cociente albúmina/creatinina: $0,6 \mu \mathrm{g} /$ mgr. Glucosa orina $5567 \mathrm{mg} / \mathrm{dL}$. Metilcetona en orina $50 \mathrm{mg} / \mathrm{dL}$. HbA1c 16,2\%. Péptido C basal $166 \mathrm{pmol} / \mathrm{L}$ (298-2350). Anticuerpos anti-GAD 0,2 KU/L (0-0,9), anti-IA2 0,2 KU/L (0-1) y anti-insulina (IAA) $77,2 \mathrm{nU} / \mathrm{mL}(\mathrm{N}<40)$. Anticuerpos IgA antitransglutaminasa $0,82 \mathrm{KU} / \mathrm{L}(0-10)$. Anticuerpos anti-TG 149 (0-40) y anti-TPO 13,3 (0-35). TSH 3,1

Exploración ginecológica: útero bicorne.

Exploración del fondo de ojo y retinografía normal.

TC abdominopélvico con contaste: agenesia dorsal del páncreas.

ECO abdominal: en riñón izquierdo múltiples quistes corticales de tamaño menor de $1 \mathrm{~cm}$, difusos por todo el parénquima renal izquierdo sin evidencia de dilatación del sistema pielocalicial ni otros hallazgos. Riñón derecho normal. Ausencia de cuerpo y cola pancreática.

Cultivo de exudado del panadizo creció Klebsiella oxytoca y el cultivo vulvovaginal fue positivo para Candida albicans.

Con la sospecha de diabetes tipo MODY se solicitó estudio genético mediante PCR semicuantitativa fluorescente, detectándose delección en heterozigosis de, al menos, los exones del 1 al 8 del gen HNF1 $\beta$ que se asocia al tipo 5 .

Se comenzó tratamiento con insulina glargina e insulina aspart $1,3 \mathrm{U} / \mathrm{kg}$. Se administró fluconazol vía oral $100 \mathrm{mg} /$ día (10 días) y un óvulo vaginal en dosis única de clotrimazol $400 \mathrm{mg}$, desapareciendo las lesiones micóticas.

En los controles realizados posteriormente se detectan niveles de HbA1c menores del 6\% 
con disminución progresiva de insulina hasta 0,5 $\mathrm{U} / \mathrm{kg}$ a los 6 meses del ingreso. A los ocho meses desapareció la dermopatía diabética y a los 18 meses del ingreso, la paciente ha recuperado el peso inicial y los requerimientos de insulina se mantienen en $0,5 \mathrm{U} / \mathrm{kg}$. La función renal se ha mantenido dentro de límites normales y microalbuminuria negativa. Únicamente mantiene un ligero aumento de transaminasas.

\section{DISCUSIÓN}

Se presenta este caso de diabetes MODY-5 por ser un tipo de diabetes monogénica muy poco frecuente con curso clínico diferente al descrito en los escasos pacientes resgistrados en la literatura ${ }^{5-7}$.

Esta enfermedad se debe a una alteración genética que condiciona una disfunción progresiva de la célula $\beta$ pancreática que le impide la correcta secreción de insulina. En nuestra paciente es de destacar la estabilidad de la enfermedad durante los cinco años posteriores al diagnóstico y la rápida evolución en los dos meses previos al ingreso con una drástica disminución de insulina que llevó a precisar insulina exógena para controlar la marcada hiperglucemia.

Hay fuentes que señalan que en este tipo de diabetes existe, junto a una disfunción progresiva de la célula $\beta$, una cierta resistencia hepática a la insulina, con hiperuricemia, hipertrigliceridemia y niveles bajos de HDL-colesterol, datos que no observamos en nuestra paciente. Sin embargo, se observa la presencia de múltiples quistes renales y útero bicorne, algo muy característico de MODY-5. Se han descrito en estos pacientes múltiples anomalías renales que son reconocibles incluso antes del nacimiento, como son displasia renal, enfermedad glomerulocística, oligomeganefronia y característicamente los quistes renales $^{5}$. De hecho, en dos series publicadas de pacientes con diabetes MODY-5, se observa en el 70-97\% alteraciones renales, $86 \%$ déficit subclínico de páncreas exocrino, $85 \%$ aumento de enzimas hepáticos, $60-83 \%$ atrofia pancreática y en el $40-80 \%$ anomalías del tracto genital como útero bicorne, agenesia bilateral del conducto de- ferente, quistes de epidídimo e infertilidad por azoospermia ${ }^{6,7}$.

Una de las funciones de HNF-1 $\beta$ es la regulación de la expresión génica en tejidos como el riñón. En este órgano, el promotor proximal del gen Pkhd1 tiene un sitio de unión para HNF-1 $\beta$, por lo que las mutaciones en este factor de transcripción inhiben la expresión de HNF-1 $\beta$ y conducen a la formación de quistes. Esto es lógico, puesto que las mutaciones del gen Pkhd1son responsables de la forma autosómica recesiva de la enfermedad renal poliquística ${ }^{8}$.

Las mutaciones en HNF-1 $\beta$ pueden ser de dos tipos, puntuales en el $60 \%$ de los casos o corresponder a delecciones grandes en el gen en el otro $40 \%$ restante. Estas mutaciones se pueden heredar con carácter autosómico dominante y verse con frecuencia afectadas varias generaciones o aparecer de novo por mutación espontánea, como parece ser el caso de nuestra paciente, puesto que los padres y hermanos no se encuentran afectos de diabetes y el resto de parentesco se desconoce por residir en Ucrania. Se ha observado que en los pacientes con grandes delecciones génicas son más frecuentes las mutaciones de novo, tienen más síntomas al diagnóstico, menos peso y requieren más dosis de insulina. Por el contrario, los pacientes con mutaciones puntuales tienen mayor edad al diagnóstico y cuando desarrollan la enfermedad renal y suelen asociarse con un fenotipo restringido a un simple órgano ${ }^{9}$.

Los casos descritos de diabetes MODY5 se asocian con frecuencia a complicaciones microangiopáticas tempranas del tipo retinopatía, neuropatía e insuficiencia renal crónica rápidamente progresiva, datos que no constatamos en nuestra paciente.

Por otra parte, es infrecuente observar extensas zonas de dermopatía diabética tanto en zona pretibial, muslos, antebrazos y hombros como se vio en esta paciente (Figs. 1, 2). El mecanismo etiopatogénico de esta dermopatía no se conoce bien, pero varias fuentes lo atribuyen a la existencia de microangiopatía, con aumento de permeabilidad vascular, asociándose a retinopatía y neuropatía ${ }^{10}$. 
Figura 1. Dermopatía diabética en piernas.

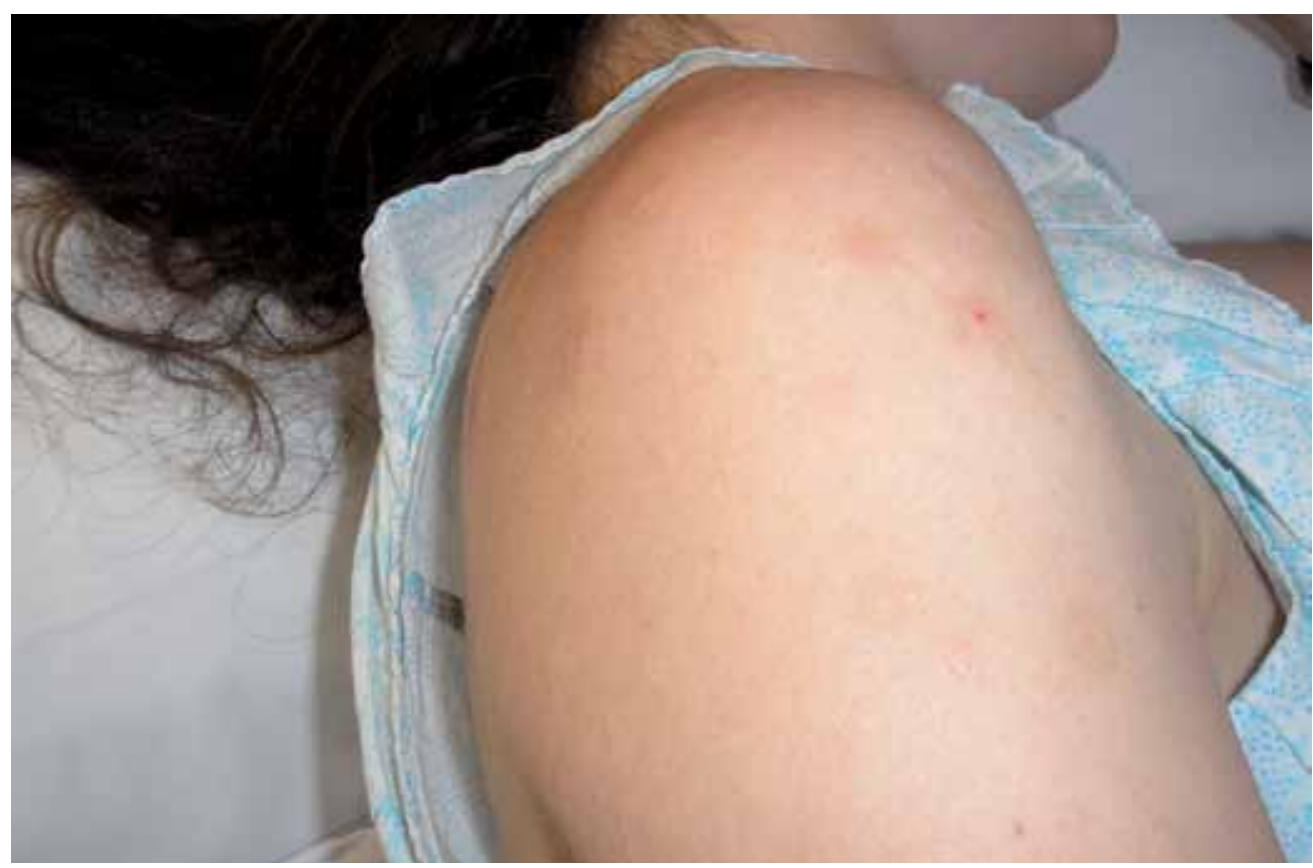

Figura 2. Dermopatía diabética en hombro. 
Por todo ello, a pesar de ser poco frecuente este tipo de diabetes, está justificado hacer pruebas de detección para las mutaciones en HFN-1 $\beta$ en diabéticos no obesos, sobre todo, cuando existen malformaciones renales o genitales asociadas, sin tener en cuenta los antecedentes familiares.

\section{BIBLIOGRAFÍA}

1. Imasaki N, Ogata M, Tomonaga O, Kuroki H, KaSAHARA T, YANO N. Liver and kidney function in Japanese patients with maturity-onset diabetes of the young. Diabetes Care 2001; 21: 2144-2148.

2. Nishigori H, Yamada S, Kohama T, Tomara H, Sho K, HonikaWA Y. Frameshift mutation, A263fsinsGG, in hepatocyte nuclear factor-1beta gene associated with diabetes and renal dysfunction. Diabetes 1998; 47: 1354-1355.

3. SÁnchez-Reyes L, Fanghänel G, Márquez-Cid ME, Salazar R, Labastida-Sánchez C, Solís-Pérez A et al. Actualización en los diferentes subtipos de diabetes tipo "MODY". Endocrinol Nutr 2001; 9: 5-11.

4. Fajans SS, Bell GI, Polonsky KS. Molecular mechanisms and clinical pathophysiology of maturity-onset diabetes of the young. N Engl J Med 2001; 345: 971-980.

5. BARRIO R. Diabetes monogénicas: enfoque diagnóstico y tipos más frecuentes. Av Diabetol 2007; 23: 333-340.

6. Bellanné-Chantelot C, Chauveau D, Gautier JF, Dubois-Laforge D, Clauin S, Beaufils S et al. Clinical spectrum associated with hepatocyte nuclear factor-1beta mutations. Ann Intern Med 2004; 140: 510-517.

7. Velho G, Bellanné-Chantelot C, Timsit J. Heterogeneidad de MODY y conducta clínica. ¿Diferentes genes como guía de distintos enfoques? Endocrinol Nutr 2004; 5 (Suppl. 2): $22 \mathrm{~S}-30 \mathrm{~S}$

8. Hiesberger T, Bai Y, Shao X, McNally BT, Sinclair AM, TIAN X, et al. Mutation of hepatocyte nuclear factor-1-beta inhibits Pkhd1 gene expression and produces renal cyst in mice. $\mathrm{J}$ Clin Invest 2004; 113: 814-825.

9. Bellanné-Chantelot C, Clauin S, Chauveau D, Collin P, Daumont M, Doulllard C, et al. Large genomic rearrangements in the hepatocyte nuclear factor-1beta (TCF2) gene are the most frequent cause of maturity-onset diabetes of the young type 5 . Diabetes 2005; 54 : 3126-3132.

10. Morgan AJ, Schwartz RA. Diabetic dermopathy: A subtle sign with grave implication. J Am Acad Dermatol 2008; 58: 447-451. 